\title{
EchoGéo
}

$47 \mid 2019$

Nouvelles géographies de la collecte

\section{Normaliser le sauvage : l'expérience française des cueilleurs professionnels}

Claire Julliand, Florence Pinton, Raphaële Garreta et Jean-Paul Lescure

\section{OpenEdition}

\section{Journals}

Édition électronique

URL : https://journals.openedition.org/echogeo/16987

DOI : $10.4000 /$ echogeo.16987

ISSN : 1963-1197

Éditeur

Pôle de recherche pour l'organisation et la diffusion de l'information géographique (CNRS UMR 8586)

Référence électronique

Claire Julliand, Florence Pinton, Raphaële Garreta et Jean-Paul Lescure, « Normaliser le sauvage l'expérience française des cueilleurs professionnels », EchoGéo [En ligne], 47 | 2019, mis en ligne le 21 avril 2019, consulté le 10 août 2021. URL : http://journals.openedition.org/echogeo/16987 ; DOI : https://doi.org/10.4000/echogeo.16987

Ce document a été généré automatiquement le 10 août 2021.

EchoGéo est mis à disposition selon les termes de la licence Creative Commons Attribution - Pas d'Utilisation Commerciale - Pas de Modification 4.0 International (CC BY-NC-ND) 


\title{
Normaliser le sauvage : l'expérience française des cueilleurs professionnels
}

\author{
Claire Julliand, Florence Pinton, Raphaële Garreta et Jean-Paul Lescure
}

\section{Introduction}

Il serait vain de prétendre décrire le monde disparate et mouvant des cueilleuses et des cueilleurs de plantes sauvages. Après avoir été longtemps associées au monde paysan, les pratiques de cueillette ont été réinvesties dans les années 1970 par de nouveaux acteurs (Larrère et la Soudière, 1985), qui y trouvaient l'occasion d'habiter un espace rural en déprise, de rompre avec un mode de vie, ou encore d'inventer de nouvelles solidarités. Les plantes sauvages qu'ils récoltent sont alors commercialisées dans le cadre de filières artisanales (tisanes, huiles essentielles, sirops, baumes) associées à des marchés de proximité, mais aussi dans les secteurs industriels (pharmacie, cosmétique, compléments alimentaires) dont elles constituent la matière première. Les années 1990-2000 marquent une nouvelle inflexion de la pratique. Les discours autour de la valeur économique de la biodiversité comme les promesses d'innovation en matière d'ingrédients naturels, modifient le regard porté sur la flore sauvage et les cueillettes commerciales. La forte croissance de la demande se traduit par une diversification et une intensification des prélèvements et pose la question de la vulnérabilité de la flore. C'est dans ce contexte de pressions multiples qu'est née en 2011, l'Association Française des professionnels de la Cueillette de plantes sauvages (AFC).

2 Nous proposons ici de discuter du renouveau des cueillettes commerciales à partir de ces points focaux que constituent la création de l'AFC puis le projet FloreS (encadré 1). FloreS est un dispositif de recherche participative ${ }^{1}$ formalisant les collaborations initiées depuis plusieurs années autour de la structuration de la profession, entre une équipe de chercheurs ${ }^{2}$ et un groupe de cueilleurs s'intéressant aux enjeux de leur métier et aux modalités de sa reconnaissance. Il s'agit aussi pour les cueilleurs de se 
fédérer autour de la nécessité d'être reconnus par les pouvoirs publics et le secteur privé en tant que co-gestionnaires de la nature, sans provoquer un excès de réglementation et de normes qui mettrait en péril leur propre activité. Le dispositif mis en place a pour objectif d'accroitre la capacité des cueilleurs à agir en tant que collectif, tout en bénéficiant du rôle d'animation et de médiation que nous avons endossé tout au long de ce projet. À travers le processus d'élaboration de la charte de l'association et de son guide de bonnes pratiques, Flores doit en particulier faciliter les échanges d'expériences et de valeurs autour du métier de cueilleur et faire émerger les points de friction comme les consensus. Nos compétences scientifiques dans le champ socioenvironnemental associées à nos expériences partagées, nous ont permis d'instaurer un principe de co-production de connaissances sur les pratiques de cueillette et l'écologie des plantes cueillies.

Après avoir rappelé les principales caractéristiques de la cueillette contemporaine et l'hétérogénéité du monde des cueilleurs dans une première partie, nous montrerons, en nous appuyant sur la genèse de l'AFC et les ambitions de ceux qui la portent, les ressorts de cette « sortie du bois ». Puis nous nous attarderons sur FloreS et sur ce qu'il donne à comprendre de ce collectif de professionnels en devenir. Au fil de nos expériences partagées, les cueilleurs ont souvent souligné que faire ce métier, c'est avant tout aimer être dehors dans un contact renouvelé avec les éléments, accepter d'être tributaire des aléas du "sauvage", faire preuve d'intuition et prendre conscience de cette coexistence avec les non-humains. Cette dimension de la relation sensible au vivant dans le métier, la manière dont elle agit sur les pratiques et sa mise à l'épreuve par le processus de professionnalisation est développée en dernière partie.

\section{De la persistance des cueillettes}

4 Pendant longtemps, le cueilleur de plantes sauvages a été confiné à la figure du paysan soumis aux aléas de la nature comme à ceux du marché. On cueillait pour soi à des fins domestiques, ou pour vendre. Progressivement, une partie importante des produits collectés va alimenter les industries naissantes via des négociants-collecteurs qui sillonnent les campagnes. Mais cette période d'essor des cueillettes commerciales sera brève du fait d'un marché en perte de vitesse et de nouvelles façons de consommer et de se soigner ${ }^{3}$ (Larrère et la Soudière, 1985). La mutation du monde agricole engagée depuis les années 1960, suivie de la recomposition des espaces ruraux et des modes d'habiter à partir des années 1980 (Hervieu et Viard, 1996), met fin à la complémentarité qui s'opérait entre pratiques agricoles et pratiques de cueillette. Avec la diffusion du modèle productiviste, l'effacement du paysan laisse une place vacante dans les campagnes que de nouvelles vocations et de nouveaux acteurs, intéressés par les usages populaires des végétaux, saisissent comme une manière de s'ancrer dans le monde rural tout en participant à la sauvegarde d'un patrimoine culturel en voie de disparition (Lieutaghi, 1983). Par le choix de vie qu'ils incarnent, ces néoruraux entrent en dissidence avec le monde moderne, pour s'installer à sa marge et se réinsérer dans la nature (Léger et Hervieu, 1983). Parallèlement, le déploiement progressif des politiques de gestion de la nature inscrit dans le sillage des mutations du monde rural, favorise l'émergence d'une profession - les gestionnaires des espaces naturels - qui s'attache à définir ce qu'il convient de protéger ou de considérer comme un patrimoine naturel, instituant ainsi d'autres points de vue qui font sortir la nature du domaine domestique 
et privé en la publicisant. Modernisation agricole, exode rural et politiques de l'environnement ne vont cesser de bouleverser le profil des cueilleurs de plantes sauvages et les conduites de cueillette dans des espaces délaissés par l'agriculture mais de plus en plus disputés.

Il n'existe pas de recensements, de statistiques ou d'évaluations globales de l'activité de cueillette à l'échelle nationale. Les cueilleurs forment un groupe hétérogène, tant du point de vue des pratiques, des volumes collectés, des territoires exploités que des circuits de commercialisation. Nous estimons que parmi ceux qui se caractérisent par de petites productions issues de cueillettes et de cultures et écoulées majoritairement en vente directe, environ trois cents appartiennent au syndicat SIMPLES ${ }^{4}$ dont les produits sont tous certifiés. On identifie également une centaine de cueilleurs œuvrant au sein de coopératives et quelques dizaines d'indépendants répondant directement aux commandes de l'industrie. Si l'ensemble de ces groupes est représenté dans le dispositif FloreS, une autre figure, celle du cueilleur occasionnel peu qualifié - salarié ou payé à la tâche - travaillant pour des collecteurs est absente. Elle échappe également à toute mise en chiffre, le système de collectage étant peu documenté ${ }^{5}$ en France depuis "Cueillir la montagne " (Larrère et la Soudière, 1985). Le collecteur ou leveur est celui qui rassemble la production de particuliers en recherche d'un revenu d'appoint ou organise des équipes formées d'une main d'œuvre souvent étrangère.

Un récent inventaire (Lescure et al., 2018) réalisé dans le cadre de Flores fait état d'un peu plus de 700 espèces végétales faisant l'objet de prélèvements à buts commerciaux dans les milieux plus ou moins anthropisés du territoire métropolitain, à l'exception des milieux urbanisés non pris en compte ${ }^{6}$. Cette biodiversité végétale utile et utilisée correspond à un peu plus de $10 \%$ de la biodiversité végétale de la métropole. Cependant, ce n'est qu'une centaine d'espèces qui sont fréquemment récoltées. Quant aux quantités réellement prélevées et aux chiffres d'affaire générés, les données appartiennent aux entreprises productrices et utilisatrices qui les gardent sous le sceau de la confidentialité.

7 Si les cueillettes se maintiennent en tant que pratiques anciennes, cette manière singulière de produire en ayant recours au "sauvage » ne rend plus indispensable l'accès au foncier agricole, contrôlé par la profession agricole depuis l'instauration de la cogestion en France (Sencébé et al., 2013). La majorité des cueilleurs que nous avons rencontrés, sont de fait des hors cadre familiaux ${ }^{7}$, à l'image et aux trajectoires assez semblables de ceux que l'on appelle les «nouveaux agriculteurs». S'ils peuvent posséder un peu de foncier, ils peuvent aussi s'en détacher. Pour caractériser sociologiquement les cueilleurs-producteurs ${ }^{8}$, nous avions exploré l'hypothèse que les dynamiques observées dessinaient les contours d'une néo-paysannerie engagée dans la lutte pour l'autonomie et la maitrise de ses savoirs (Pinton et al., 2015). On peut également formuler l'hypothèse que l'attachement à la terre et à sa transmission familiale (échelle de l'exploitation agricole), fondement de l'identité sociale de l'agriculteur depuis la révolution française (Pibou, 2016), n'est pas transposable aux cueilleurs. On observe parmi ces derniers un déplacement identitaire à l'échelle de la plante, du site de cueillette et du territoire, que l'on peut nommer de façon plus totalisante la biodiversité. Ce qu'il importe alors de transmettre, ce n'est plus tant la terre qu'une façon de l'habiter, un mode opératoire et les savoirs associés. transmission du savoir au sein de la famille et pas d'institution dédiée à la formation, et 
quelles compétences acquérir? Nous avons pu constater, au moins chez les producteurs-cueilleurs SIMPLES, que l'accès aux savoirs est un champ d'activité sociale extrêmement structurant où l'expérience de l'individu domine (Pinton et al., 2015). Les processus d'acquisition se font dans des cadres souvent peu institutionnalisés, ce qui donne au compagnonnage et à l'autoformation une place importante. Si l'essentiel du métier s'acquiert au cours de stages avec des cueilleurs installés, des formations sont néanmoins dispensées et validées par quelques centres de formation professionnelle et de promotion agricole (CFPPA). De nombreuses formations en interne (bien qu'ouvertes sur l'extérieur) sont aussi organisées. Généralement éligibles aux Fonds pour la formation des entrepreneurs du vivant (VIVEA), elles sont prisées par les producteurscueilleurs des filières artisanales, dont les connaissances scientifiques initiales dépendent du niveau de formation d'origine, et pour lesquels l'apprentissage du métier passe aussi par un approfondissement des connaissances sur les plantes ${ }^{9}$.

9 La volonté de nombreux cueilleurs de se structurer à petite échelle a été perceptible assez rapidement à travers la création d'organisations, de réseaux, ou de structures professionnelles souvent implantées dans les zones de moyenne montagne ${ }^{10}$. Néanmoins, du fait d'une économie longtemps perçue comme informelle, souterraine ou anecdotique, les activités des cueilleurs sont demeurées longtemps marginales pour les services de l'État, qu'ils travaillent à l'encadrement agricole ou pour la protection de la nature.

\section{Sortir du bois pour défendre une certaine vision du métier}

10 Nous l'avons déjà évoqué, les façons d'être cueilleur sont multiples. Certains se disent simplement " cueilleur ", d'autres " producteur-cueilleur ", d'autres encore " cueilleurherboriste » ou "paysan-cueilleur " et même, dans un autre registre "paysan sans terre ». Dans ces autodéfinitions qui confinent parfois à la revendication, se dit la diversité des activités et des vues que chacun porte sur son métier. Sous des formes revisitées par le temps, se perpétuent la dispersion, la fluctuation et l'enchevêtrement des intérêts matériels et des investissements symboliques qui semblaient inhérents aux cueillettes (Larrère et la Soudière, 1985).

11 Cependant c'est bien de métier dont les cueilleurs de l'AFC veulent parler désormais. En effet, alors même que la cueillette ne bénéficie d'aucun statut professionnel spécifique, plusieurs d'entre eux estiment qu'il serait important de pouvoir faire la distinction entre ceux qu'ils qualifient de "non professionnels ", cueilleur saisonnier ou à la tâche approvisionnant des collecteurs "sans aucun souci de préservation des sites» et des "professionnels» qui seraient conscients des enjeux de gestion des ressources végétales sauvages. En phase avec les préoccupations écologiques du moment où les alertes sur la régression de la flore sauvage en France dans sa diversité et sa répartition spatiale sont de plus en plus prégnantes, cette vision idéalisée des cueillettes commerciales oppose ceux qui assurent et assument une pratique gestionnaire vis-à-vis de la ressource et les «autres", auteurs de "pillages » et de "razzias " qui mettent localement à mal des stations, voire même la présence d'espèces (Garreta, 2015). Les services de l'État ont plutôt vent des pratiques des seconds. C'est par leurs excès et leurs dérives, ou à travers les craintes qu'elles pouvaient susciter chez les naturalistes, que les cueillettes ont d'abord été appréhendées par les autorités en charge de 
l'environnement. C'est donc dans une logique de protection - voire de restauration qu'une cinquantaine d'espèces ont été sélectionnées à dire d'expert dans la liste annexée à un arrêté de $1989^{11}$ qui permet aux préfets d'interdire ou de réglementer localement la cueillette d'une ou plusieurs d'entre elles en fixant les dates et les modalités d'application des mesures mentionnées.

La réflexion autour des cueillettes ne s'est pas arrêtée à la mise en place de cet outil réglementaire spécifique. Souhaité par le Ministère de l'Environnement, le Guide technique de la cueillette des plantes sauvages paraît en 1992 (Pointereau et Galland, 1992). Plusieurs cueilleurs professionnels sont associés à cet ouvrage. A partir de leurs observations et expériences, ils livrent des préconisations générales de gestion de la ressource et des techniques particulières appliquées à treize espèces choisies. Si ce guide fut peu diffusé, les jalons d'une réflexion étaient bel et bien posés. Au cours des décennies suivantes, de nouvelles initiatives de concertation pour prendre en compte le savoir des cueilleurs, ont vu le jour mais sont restées peu coordonnées. Pendant ce temps, les lignes des politiques publiques aux échelles internationales et nationales en faveur de la biodiversité12 ont bougé et invitent à un changement de perspective, considérant la valorisation des plantes par le marché comme une opportunité de garantir leur conservation. En France, certaines modalités d'accès et de valorisation économique de la flore sauvage sont redéfinies par la «loi pour la reconquête de la biodiversité de la nature et des paysages » qui prévoit d'adapter la règlementation de la cueillette en la modifiant (Cambecèdes et Garreta, 2018).

Cette expansion de l'économie du «sauvage» qui touche l'ensemble des pays européens suscite l'élaboration de nouvelles normes en matière de traçabilité et durabilité par les entreprises en aval des filières qui se sont elles aussi multipliées et diversifiées. Les pratiques de cueillette sont appelées à se transformer face à un environnement, politique, réglementaire et institutionnel en pleine évolution. Faisant le constat de ces mutations, désireux de se démarquer des « mauvaises pratiques » qui entachent l'image du métier et soucieux de défendre leurs intérêts intimement corrélés à la pérennité de la ressource sauvage, une cinquantaine de cueilleurs ont décidé de se fédérer pour se faire entendre d'une seule et même voix par les pouvoirs publics. À l'automne 2011, l'Association Française des professionnels de la Cueillette des plantes sauvages dépose donc ses statuts avec l'objectif de représenter et promouvoir le métier et de diffuser de bonnes pratiques pour une cueillette respectueuse « des ressources, des milieux et des hommes $»^{13}$. La mise en avant de leurs savoirs et savoir-faire relatifs à la flore sauvage s'inscrit donc au cœur du processus de structuration (Garreta et Julliand, 2017). Le tour de force de la jeune association est d'avoir réuni autour de l'idée de gestion de la ressource - et de son accès - des sensibilités différentes qui jusqu'alors se pensaient contraires. Le lien particulier à la nature, au vivant, et l'importance accordée aux gestes professionnels se sont montrés prépondérants dans cette construction (Albert-Llorca et Garreta, 2016).

Aujourd'hui, les cueilleurs de l'AFC sortent de l'ombre. Avec eux, c'est toute l'économie des cueillettes, les questions de responsabilité qu'elles posent et la dimension nationale de leur régulation qui sont mises en avant. Le projet FloreS va permettre la mise en débats de ces sujets au sein du jeune collectif. 


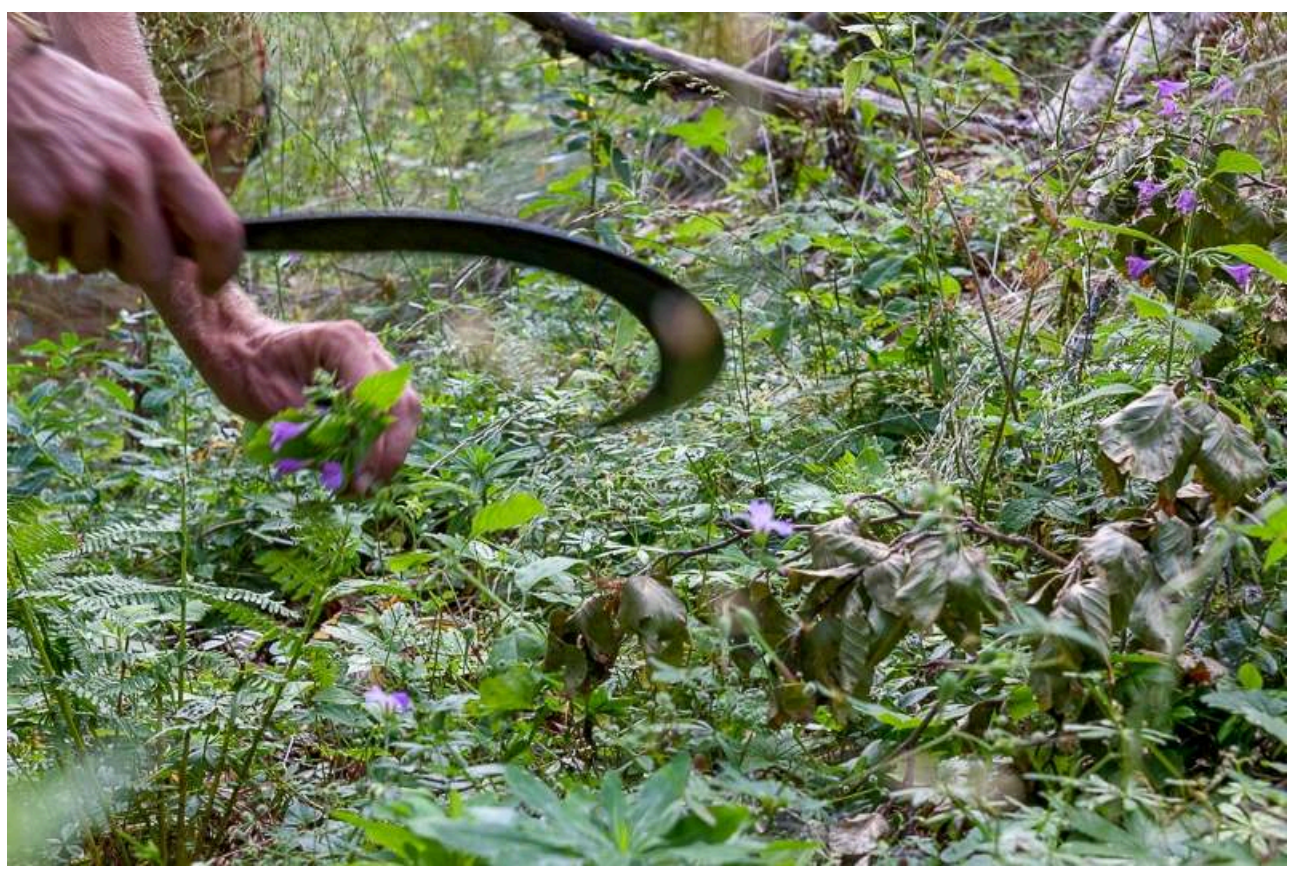

«Il faut aimer ces moments-là où tu cueilles, sinon c'est mort. J'embauche des gens super branchés par la nature, mais au bout de dix minutes ils disent : on ne va pas faire ça huit heures ! En fait la base comme moi je le vis, c'est les heures passées avec tes bourgeons ou avec ta serpette, avec ta faucille, avec ta pioche, c'est un boulot de gestes. »

Auteur: Marion Lavabre/CBNPMP.

\section{FloreS, un lieu d'expérimentation des aspirations de la profession}

15 L'AFC constituée, un projet de charte s'est rapidement imposé comme la première action à mettre en œuvre pour s'engager dans la reconnaissance de la profession. Il est également apparu qu'il serait sans doute nécessaire d'adosser à ce code éthique d'autres outils, voire d'envisager à terme une démarche de certification des produits issus de cueillettes durables ${ }^{14}$.

Pour poursuivre cette réflexion, l'AFC devait créer un espace d'échanges et de débats pour socialiser les expériences individuelles et la diversité des pratiques de cueillette. Si les membres de l'AFC, se considèrent comme des professionnels à part entière, d'aucun n'avait le sentiment d'appartenir à une profession, en tant que groupe de pairs clairement identifié. La charte est alors apparue comme un projet potentiellement fédérateur, apte à soutenir l'émergence d'une identité sociale partagée nécessaire pour gagner en visibilité et lisibilité vis-à-vis de l'extérieur.

17 L'histoire de l'AFC n'est pas faite que d'un entre soi. Elle est fortement liée à l'engagement, depuis les années 2000, de personnes issues des milieux de la recherche scientifique et de la conservation de l'environnement et qui ont participé au renouvellement de l'intérêt porté aux activités de cueillette commerciale par les structures en charge des questions environnementales. De terrain en terrain, ces soutiens ont été progressivement embarqués dans une dynamique associative et ont adopté une démarche dialogique avec les cueilleurs. Un appel à projet de la Fondation 
d'entreprise Hermès $(\mathrm{FeH})$ sur le thème Biodiversité et savoirs locaux allait leur permettre avec d'autres collègues ${ }^{15}$ d'aller plus avant dans leur implication. La sélection par la FeH du projet intitulé Flores (encadré 1), leur a permis d'accompagner l'AFC dans ses missions tout en menant une réflexion plus générale sur les modalités d'une valorisation durable de la flore sauvage en France métropolitaine avec une attention particulière accordée aux rapports entre savoirs locaux et normes de qualité.

Flores, conçu sur un mode participatif et réflexif, a été imaginé pour aider l'AFC à se structurer et à mettre en œuvre ses missions. Si les participants se connaissent pour la plupart individuellement, ils se trouvent engagés dans une configuration inédite. Le projet va induire un certain formalisme qui va se révéler être une nouveauté pour l'association. Sa conduite est alors marquée par un mélange de familiarité et de distance. L'AFC y est représentée par six membres impliqués dans le comité de pilotage et par un groupe de sept cueilleurs référents, tous volontaires. Bien que le projet ait été pensé comme terrain d'expérience du collectif, les charges et responsabilités à assumer reposent donc sur un nombre restreint de membres de l'association.

Ces quatre années de rencontres, d'échanges et de travaux - des ateliers thématiques réunissant un plus large public aux séances de travail en petit comité du groupe de cueilleurs référents - ont nécessité des apprentissages réciproques dont on ne rendra pas compte ici. Il a fallu apprendre à se connaître et à se coordonner entre chercheurs et cueilleurs mais aussi entre cueilleurs. Un langage commun a dû s'élaborer pour travailler ensemble aux outils choisis par l'AFC pour construire la reconnaissance de la profession. Ces épreuves ont finalement participé à la trajectoire de consolidation de l'AFC et à la constitution d'une vision réaliste de ce que signifie entrer dans un processus de normalisation.

\section{Encadré 1 - Le projet FloreS}

FloreS - Valoriser durablement la flore sauvage en France métropolitaine. Une recherche-participative qui accompagne les professionnels de la cueillette de plantes sauvages dans la reconnaissance de leurs savoirs et savoir-faire et la définition de bonnes pratiques (2014-2018)

Porteur : Institut de géographie et durabilité de l'Université de Lausanne (Igd, Unil)

Partenaires scientifiques et techniques : AgroParisTech (UPS), Conservatoire botanique national des Pyrénées et de Midi-Pyrénées (CBNPMP), Conservatoire National des Plantes à Parfum, Médicinales, Aromatiques et Industrielles (CNPMAI)

Financements : Fondation d'entreprise Hermès et apports complémentaires de l'Unil, du Ministère en charge de l'environnement et de FranceAgriMer.

\section{Les réalisations :}

Une charte de l'AFC portant les principes et les valeurs portés par les professionnels ;

Le canevas d'un référentiel professionnel appelé « Guide de bonnes pratiques » (GBP) avec ses volets « recommandations générales » et « livrets techniques » devant faciliter la mise en œuvre des engagements énoncés dans la charte ; Cinq modèles de livrets techniques correspondant à différents scénarios de 
cueillette ;

Un itinéraire méthodologique pour la co-construction des contenus du GBP.

Son objectif majeur est de proposer des éléments de méthodologie opérationnelle à destination des professionnels de la cueillette. La charte et le GBP avec ses livrets techniques sont considérés comme des outils pouvant à la fois sensibiliser l'ensemble des cueilleurs et contribuer à la reconnaissance de la profession par l'État et les institutions publiques en charge des questions environnementales ou agricoles et les autres opérateurs économiques des filières utilisatrices d'ingrédients naturels.

FloreS s'est déployé principalement autour de trois ateliers thématiques menés de manière participative et avec l'appui de spécialistes des problématiques abordées. Ces temps de rencontre ont été respectivement consacrés à l'identité et à l'éthique professionnelle, aux normes de qualité et leur pertinence pour développer une cueillette durable et enfin aux aspects d'encadrement et de régulation de l'activité en lien avec les acteurs de la gestion et de la conservation de la flore et des espaces naturels. Le groupe de cueilleurs référents formé lors du $1^{\mathrm{er}}$ atelier est composé de sept professionnels aux profils et aux pratiques variés. Il est chargé de représenter les membres de l'AFC lors des ateliers et de travailler à l'élaboration des différents outils retenus par le collectif pour construire la reconnaissance de la profession.

Extrait de https://wp.unil.ch/flores/

\section{L'expérience sensible avec le végétal est-elle soluble dans la professionnalisation?} d'attachement des cueilleurs au vivant de manière générale et au végétal en particulier et la manière dont ils engagent ces liens dans la construction des identités sociales et professionnelles individuelles et collectives. L'expérience sensible avec le végétal et les autres composantes du territoire de cueillette est reconnue par les cueilleurs comme agissante dans leur activité en tant que source de compréhension de ce qui se joue et facteur de transformation des pratiques. Elle est également convoquée dans les discours pour renforcer l'appartenance au collectif et pour légitimer certaines postures comme celles de co-gestionnaire et de sentinelle de la nature. Pourtant, il reste difficile de garder cette dimension vivante dans le processus de professionnalisation, notamment dans la formalisation des contenus des outils. En rendre compte comme une forme de savoir, la traduire en mots reste un exercice difficile voire impossible.

Si l'adhésion à ce métier repose en partie sur « le plaisir à être dans la nature " associé à un fort sentiment de liberté, la viabilité de l'activité est intrinsèquement liée aux conditions même d'existence de la flore et des milieux naturels. Aussi, exercer «durablement » ce métier, c'est chercher à s'inscrire dans une forme de réciprocité, faire l'expérience d'une médiation avec le vivant.

«Je me considère donc comme un prédateur comme les autres, mais j'essaie toujours de me penser en tant que cueilleur, de tenter de comprendre comment on doit cueillir [...]. Je me dis : Eh bien, essaie de regarder les autres êtres qui mangent des plantes, des feuilles, des bourgeons... essaie de t'en inspirer, de voir comment ils font... parce qu'eux, semblent être beaucoup plus en accord, beaucoup moins 
violents que nous avec l'environnement. » (Thévenin, 2017 - membre de l'AFC et de Flores).

22 En effet, la cueillette est couramment décrite comme une activité de prédation au même titre que la chasse ou la pêche (Dalla Bernardina, 2012) dont la "réussite » dépend étroitement de la disponibilité des ressources, de leur accessibilité, et à plus long terme de leur pérennité. Réussir une cueillette en tant que professionnel ne se limite donc pas tant à la possibilité d'accéder une seule et unique fois à une station de cueillette que de pouvoir déployer des manières de faire, de coexister avec le vivant qui permettent de sécuriser - dans les limites de ce qui est contrôlable - l'accès aux sites de cueillette. Cette perspective de durabilité - à savoir prélever sans endommager la capacité de renouvellement des espèces en présence et de la ressource en particulier place le cueilleur dans une situation qui l'amène à devoir négocier avec les différentes parties prenantes du lieu de cueillette, qu'ils soient humains (propriétaire, usager, contractuel, gestionnaire, etc.) ou non-humains (différentes composantes du milieu).

C'est ainsi que les professionnels de l'AFC se distinguent du « mauvais cueilleur » qui opérerait de manière opportuniste sans se soucier de l'impact de sa collecte.

Ceci étant, l'enjeu de la relation sensible au vivant et par extension celui de bonnes pratiques ne sont évidemment pas perçus de manière similaire par les cueilleurs selon que leurs activités s'inscrivent dans une démarche artisanale ou approvisionnent le secteur industriel. Certains placent d'emblée le respect du vivant au cœur de leurs valeurs et de leurs pratiques, alors que d'autres se font plus pragmatiques et affichent des objectifs de durabilité qui visent davantage à leur garantir un accès aux ressources.

De plus, si ces bonnes pratiques en tant que façons d'agir en réciprocité avec le végétal semblent assez évidentes à énoncer lorsqu'il s'agit de lister des comportements à privilégier, celles-ci deviennent plus délicates à formuler au moment de rendre compte de ce qui est concrètement mis en œuvre sur le terrain. Ainsi dans le processus de coconstruction du référentiel professionnel, il reste difficile de se référer explicitement à l'expérience sensible, c'est-à-dire ce à qu'elle produit sur l'individu et en quoi cela peut influencer ses manières de faire tant au niveau de la cueillette elle-même que de la gestion des ressources et des milieux. Les différentes mises en situation au sein de Flores (ateliers de travail, élaboration de la charte, du guide de bonnes pratiques et ses livrets techniques) ont toutes révélées ces difficultés de traduction de l'expérience sensible.

L'AFC s'engage dans la voie ambitieuse d'une éthique professionnelle où prendre soin de la ressource devient central. Le «Guide de bonnes pratiques » qui doit permettre sa mise en œuvre tente d'articuler non sans difficultés les différentes formes de savoir du métier y compris celles issues du domaine du sensible. Cette approche se différencie en ceci de celle des gestionnaires et des experts de l'environnement, qui ont conscience de ne pas détenir les connaissances suffisantes pour établir des normes qui soient à la fois légitimes et pertinentes. Par analogie avec le monde paysan, il s'agit pour les cueilleurs de retrouver (ou ne pas perdre) ce dont a été privé l'agriculteur en se professionnalisant, c'est-à-dire une proximité avec les processus naturels au profit d'un savoir savant et technique venu d'en haut. 

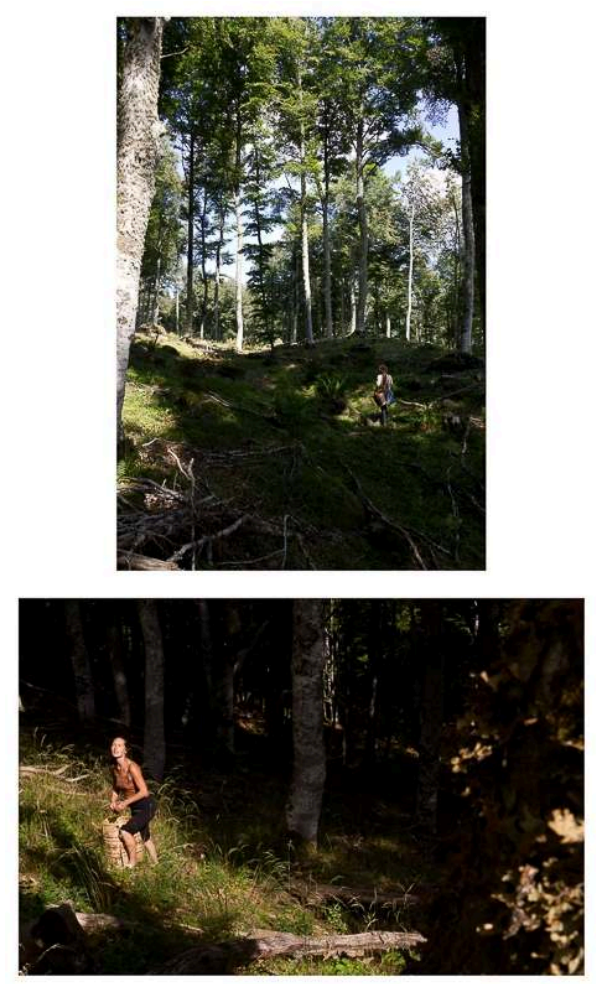

"Quand t'es toute seule tu ne fais pas de bruit, tu te fonds complètement. Y'a des fois où je me surprends à faire attention à ne pas faire de bruit quand je marche. »

« Je suis bien là où je suis, libre de mes mouvements. J'aime l'atmosphère de cette forêt et de cette cueillette. »

Auteur: Marion Lavabre/CBNPMP

\section{Conclusion}

27 FloreS a été pour les cueilleuses et les cueilleurs de l'AFC, un espace pour rechercher les voies d'une reconnaissance professionnelle et en mesurer les potentialités, les difficultés et les contradictions. Il a permis de renforcer à plusieurs niveaux le collectif, malgré l'hétérogénéité des pratiques et des formes d'organisation des professionnels. L'association a ainsi vu son nombre de membres doubler le temps de FloreS, comptant désormais une centaine de personnes. Sa légitimité auprès des autres acteurs a également progressé comme en atteste les récentes discussions pour la création d'un "Observatoire national des cueillettes commerciales" avec les pouvoirs publics ou encore les invitations à participer à d'autres projets de recherche. Malgré ces avancées, le collectif reste néanmoins structurellement fragile dans la mesure où, avec la fin de Flores, l'association perd une force d'animation et de coordination du réseau.

Il nous semble important de souligner pour conclure, que l'expérience fédératrice de l'AFC est à notre connaissance, inédite en Europe où les cueillettes commerciales se déploient principalement à travers un système de collectage où le cueilleur n'est bien souvent qu'un simple intermédiaire. Dénonçant ce système, les cueilleurs de l'AFC placent la liberté, l'autonomie et le bon usage de la flore au cœur du processus de reconnaissance de leur métier. Il serait alors intéressant de pouvoir interroger plus précisément l'activité de l'AFC en tant que mouvement social engagé dans l'horizon 
normatif de la durabilité. Ce collectif est-il le «symbole d'une résistance dans un monde [qui s'est] précarisé » (Tsing, 2017) ou est-il aussi porteur d'une conception des rapports de l'homme à son milieu en rupture avec la modernité (Latour, 2017; Tsing, 2017) parce qu'il donnerait à voir de nouvelles manières de collaborer et d'habiter avec la terre (Centemeri, 2018)?

\section{BIBLIOGRAPHIE}

Albert-Llorca M., Garreta R., 2016. Des sociétés rurales européennes aux cueilleurs professionnels de plantes sauvages : visions et pratiques de la nature. In Javelle A., Les relations Homme-Nature dans la transition agroécologique. Paris, L'Harmattan, p. 107-124.

Cambecèdes J., Garreta R., 2018. La cueillette des plantes sauvages : exploitation des ressources naturelles et conservation d'un patrimoine naturel. Journal of new sciences, Agriculture and Biotechnology, CIRS (13), p. 3355-3365.

Centemeri L., 2018. Health and the environment in ecological transition: the case of the permaculture movement. The Relationship between Environment, Health, and Disease Toward a MultiSpatial and Historical Approach, in Press. https://hal.archives-ouvertes.fr/hal-01613420/document

Dalla Bernardina S. (dir.), 2012. L'appel du sauvage. Refaire le monde dans les bois. Rennes, Presses universitaires de Rennes, $214 \mathrm{p}$.

Garreta R, Julliand C., 2017. Pour un autre regard sur la cueillette commerciale de plantes sauvages : les pratiques gestionnaires des cueilleuses et cueilleurs professionnels. In CBN Massif central, Actes des $3^{\text {èmes }}$ Rencontres végétales du Massif central : Découvrir, comprendre et protéger la flore et la végétation du Massif central, p. 155-162.

Garreta R., 2015. La gentiane jaune des Pyrénées, les racines de la discorde. In Lieutaghi P et Musset. D., Les racines ou la métaphore des origines. Forcalquier, C'est-il à dire Éditions, p. 51-162.

Hervieu B., Viard J., 1996, Au bonheur des campagnes. La Tour d'Aigues, Éditions de l'Aube, 160 p.

Larrère R., de la Soudière M., 1985. Cueillir la montagne. Lyon, La manufacture, 253 p.

Léger D., Hervieu B., 1983. Des communautés pour les temps difficiles. Néo-ruraux ou nouveaux moines. Paris, Le Centurion, $216 \mathrm{p}$.

Lescure J.-P., Thévenin T., Garreta R. et Morisson B., 2018. Les plantes faisant l'objet de cueillettes commerciales sur le territoire métropolitain. Une liste commentée. Le Monde des plantes, 517 [2015], p. 19-39.

Lieutaghi, P. 1983. Les Simples entre nature et société : histoire naturelle et thérapeutique, traditionnelle et actuelle, des plantes médicinales françaises. Mane, éditions de l'Association Études populaires et initiatives, $159 \mathrm{p}$.

Natures Sciences Sociétés, 2017. Dossier « Des recherches participatives dans la production des savoirs liés à l'environnement », vol. $25, \mathrm{n}^{\circ} 4, \mathrm{p} .325-454$.

Pibou E., 2016. Paysans de passage. Les fermiers du mouvement Terre de Liens en France. Thèse de doctorat en sociologie, Université Toulouse 2 - Jean Jaurès, 481 p. 
Pinton F, Julliand C., et Lescure J.P., 2015. Le producteur-cueilleur, un acteur de l'interstice. Anthropology of food [En ligne], S11. http://journals.openedition.org/aof/7902

Pointereau P., Galland J.P., 1992. Guide technique de la cueillette des plantes sauvages. Toulouse, Solagro, rapport, $53 \mathrm{p}$.

Sencébé Y., Pinton F., Alphandery P., 2013. Expertise foncière et contrôle des terres agricoles en France. Sociologie, vol. 4, n³, p. 251-268.

Thévenin T., 2017. Témoignage d'un cueilleur, paysan-herboriste où comment concilier éthique et pratiques. In Lieutaghi P. (dir.), Les plantes manipulées : morales du végétal ? Actes du séminaire d'ethnobotanique de Salagon. Forcalquier, Éditions Musée de Salagon, p. 208-212.

Tsing A. L., 2017. Le Champignon de la fin du monde : sur la possibilité de vivre dans les ruines du capitalisme. Paris, La Découverte/Les Empêcheurs de penser en rond, 415 p.

\section{NOTES}

1. Se reporter au dossier thématique de la revue Natures Sciences Sociétés sur les recherches participatives (2017) qui souligne le foisonnement des modalités de la participation. Nous avons fait le choix de ne pas en discuter dans cet article.

2. De diverses disciplines et rattachés à des institutions distinctes, chacun d'entre nous compte de nombreuses années d'observation du milieu des cueilleurs et des impacts de la cueillette sur la ressource. Plusieurs articles et rapports d'expertise ont été produits où se font jours les gestes et les discours des cueilleurs professionnels.

3. Au début des années 1960, on assiste à la mise en culture de certaines plantes, au déclin de l'herboristerie et au développement de l'industrie pharmaceutique avec la chimie de synthèse.

4. Syndicat Inter-Massif pour la Production et l'Économie des Simples créé en 1982.

5. Les récents travaux du CBNPMP autour des cueillettes de la gentiane jaune et de l'arnica confirment que ce système s'intéressant principalement aux ressources naturelles communes à fort rendement est en progression et s'ouvre à de nouvelles plantes.

6. Rappelons que les champignons ne font pas partie du règne végétal et sont donc exclus des cueillettes considérées.

7. Les hors cadre familiaux ne bénéficient pas d'une transmission de terres dans un cadre familial et leurs expériences témoignent des difficultés pour trouver des terres et accéder aux aides publiques.

8. Une part de l'ensemble des cueilleurs rencontrés associe la cueillette à la culture de plantes aromatiques et médicinales sur de petites surfaces, leur transformation sur place et leur commercialisation dans des circuits courts.

9. Concernant la botanique et les propriétés des plantes et leurs usages, nombreux sont les producteurs qui ont suivi des formations: Association pour le renouveau de l'herboristerie, École lyonnaise des plantes médicinales, Institut méditerranéen de documentation, d'enseignement et de recherches sur les plantes médicinales ou encore le Diplôme universitaire de phytothérapie de Bobigny, etc.

10. Comme par exemple Biotope des montagnes (1970), le syndicat SIMPLES (1982), la SICARAPPAM (1987), le GIE « Plante infuse » (1989).

11. "Arrêté relatif à la liste des espèces végétales pouvant faire l'objet d'une réglementation préfectorale permanente ou temporaire »- Arrêté ministériel du 13 octobre 1989 - R412-8 Code de l'Environnement. 
12. On peut citer la Convention sur la Diversité Biologique (CDB) ratifié par la France dès 1992, le protocole de Nagoya adopté en 2010 qui la complète, et sa déclinaison nationale (loi pour la reconquête de la biodiversité, de la nature et des paysages) adoptée en juillet 2016.

13. Site internet de l'AFC : http://www.cueillettes-pro.org/0-301-Preambule.html

14. Chaber L et al., 2013. Pré-étude du projet de charte nationale de cueillette professionnelle de plantes premiers éléments de diagnostic. Ministère de l'environnement et FranceAgriMer.

15. Valérie Boisvert (Igd -Unil), Jocelyne Cambecèdes et Béatrice Morisson (CBNPMP).

\section{RÉSUMÉS}

L'engouement pour les produits naturels redynamise à l'échelle mondiale les cueillettes commerciales de plantes sauvages. En France métropolitaine, cette activité a longtemps été perçue comme marginale alors que plus de $10 \%$ de la biodiversité végétale serait aujourd'hui concernée par cette économie du "sauvage». Soucieux de se distancier des "pillages» qui agitent parfois les territoires ruraux et d'assurer la préservation des ressources végétales, des cueilleuses et des cueilleurs se sont réunis en 2011 au sein de l'Association française des professionnels de la Cueillette de plantes sauvages (AFC). Ce jeune collectif souhaite faire reconnaitre le métier dans sa diversité et construire une éthique de la cueillette en concertation avec les acteurs des filières utilisatrices d'ingrédients naturels et les gestionnaires de l'environnement. Des collaborations avec des acteurs des milieux de la recherche et de la conservation ont permis de faire émerger une dynamique participative et réflexive pour accompagner ce processus de professionnalisation et penser les modalités de sa réalisation. A travers ce dispositif, les membres de l'AFC vont mettre à l'épreuve leurs valeurs, leurs savoirfaire et leurs rapports à la plante. En nous appuyant sur ces expériences partagées, des prémices de l'association au projet Flores, nous proposons de discuter du renouveau des cueillettes commerciales en France métropolitaine.

The growing interest for natural products is revitalising commercial wild plant gatherings worldwide. In metropolitan France, this activity has long been perceived as marginal, whereas more than $10 \%$ of plant biodiversity relates today to this "wild" economy. In 2011, gatherers founded the Association Française des professionnels de la Cueillette de plantes sauvages (AFC) to ensure the preservation of plant resources and prevent from pillages that can occur in rural areas.". This new collective wishes to have the profession recognized in its diversity and to build a harvesting ethic in consultation with the actors of the sectors using natural ingredients and the environmental managers. Collaborations with actors from the research and environmental conservation communities have made it possible to develop a participative and reflective dynamic to support this professionalization process and to develop the modalities of its implementation. Through this scheme, AFC members will test their values, know-how and relationship to the plant. Building on these shared experiences, from the beginning of the association to the Flores project, we propose to discuss the renewal of commercial wild plant harvesting in metropolitan France. 


\section{INDEX}

Mots-clés : recherche participative, cueillette commerciale, gestion de la ressource végétale, profession, bonnes pratiques, expérience sensible.

Keywords : participatory research, commercial harvesting, plant resource management, profession, good practices, sensitive experience

Thèmes : Sur le Champ - Sur le Terrain

\section{AUTEURS}

\section{CLAIRE JULLIAND}

Claire Julliand, claire.julliand@unil.ch, est coordinatrice du projet FloreS et doctorante au sein de l'Institut de géographie et de durabilité de l'Université de Lausanne. Elle a récemment publié : - Julliand C., 2017. Vers une reconnaissance professionnelle de la cueillette commerciale des plantes sauvages en France. In Lieutaghi P. (dir.), Les plantes manipulées : morales du végétal ? Actes du séminaire d'ethnobotanique de Salagon. Forcalquier, Éditions Musée de Salagon, p.218-222. - Garreta R, Julliand C., 2017. Pour un autre regard sur la cueillette commerciale de plantes sauvages : les pratiques gestionnaires des cueilleuses et cueilleurs professionnels. In CBN Massif central, Actes des $3^{\text {èmes }}$ Rencontres végétales du Massif central : Découvrir, comprendre et protéger la flore et la végétation du Massif central, p. 155-162.

\section{FLORENCE PINTON}

Florence Pinton, florence.pinton@agroparistech.fr, est professeur de sociologie à AgroParisTech, UMR SADAPT, Université Paris Saclay. Elle a, entre autres, publié

- Pinton F., Julliand C., Lescure J.-P., 2015. Le producteur-cueilleur, un acteur de l'interstice ? In Les gardiens de la terre, Anthropology of food, [En Ligne]. http://aof.revues.org/7902

- Alphandery P., Sencébé Y., Pinton F., 2016. Ce que co-habiter veut dire : fragmentation et recomposition dans les communes rurales. Ethnologie française, $\mathrm{n}^{\circ} 1, \mathrm{p} .141-184$.

- Joly N., Pinton F., 2016. Les concepts et les méthodes des sciences sociales pour la production et le partage des savoirs agronomiques. Le point de vue de la sociologie. Agronomie, environnement et sociétés. Savoirs agronomiques pour l'action, vol. 6, n², p.45-48.

\section{RAPHAËLE GARRETA}

Raphaële Garreta, raphaele.garreta@cbnpmp.fr, est chargée de mission à l'ethnologie au Conservatoire botanique national des Pyrénées et de Midi-Pyrénées. Elle a récemment publié : - Albert-Lorca M., Garreta R., 2016. Des sociétés rurales européennes aux cueilleurs professionnels de plantes sauvages : visions et pratiques de la nature. In Javel A. (dir.), Les relations Homme-Nature dans la transition agroécologique. Paris, L'Harmattan, p.107-124.

- Garreta R., 2017. Un tour d'horizon de quelques cueillettes commerciales. In Lieutaghi P. (dir.), Les plantes manipulées: morales du végétal ? Actes du séminaire d'ethnobotanique de Salagon. Forcalquier, Éditions Musée de Salagon, p. 195-201.

- Cambecèdes J., Garreta R., 2018. The harvesting of wild plants: exploitation of natural resources and conservation of a natural heritage. Journal of new sciences [En ligne], vol. spécial Agriculture and Biotechnologie, CIRS (13), p. 3355-3365. http://www.jnsciences.org/agri-biotech/77-volumespécial-conférence-cirs-2017/483-the-harvesting-of-wild-plants-exploitation-of-naturalresources-and-conservation 


\section{JEAN-PAUL LESCURE}

Jean-Paul Lescure est directeur de recherche honoraire à l'Institut de recherche pour le développement. Il a notamment publié :

- Lescure J.-P., Thévenin T., Garreta R. et Morisson B., 2018. Les plantes faisant l'objet de cueillettes commerciales sur le territoire métropolitain. Une liste commentée. Le Monde des plantes, 517 [2015], p. 19-39. 